\title{
Lapurdum
}

LAPURDUM Euskal ikerketen aldizkaria | Revue d'études basques |

Revista de estudios vascos | Basque studies review

$4 \mid 1999$

Numéro IV

\section{Le motif linguistique dans l'habitation de néo-style basque}

Pierre Bidart

\section{OpenEdition}

\section{Journals}

Édition électronique

URL : http://journals.openedition.org/lapurdum/1567

DOI : 10.4000/lapurdum.1567

ISSN : 1965-0655

\section{Éditeur}

IKER

Édition imprimée

Date de publication : 1 octobre 1999

Pagination : 279-285

ISBN : 2-84127-156-0

ISSN : 1273-3830

Référence électronique

Pierre Bidart, «Le motif linguistique dans l'habitation de néo-style basque », Lapurdum [En ligne], 4 | 1999, mis en ligne le 01 juin 2010, consulté le 29 janvier 2020. URL : http://journals.openedition.org/ lapurdum/1567 ; DOI : 10.4000/lapurdum. 1567 


\section{Pierre BIDART}

\section{LE MOTIF LINGUISTIQUE DANS L'HABITATION DE NÉO-STYLE BASQUE}

L'examen des modalités d'usage du "motif" met facilement en évidence son caractère transdisciplinaire : nombre de champs d'étude s'y réfèrent, parfois même en lui accordant une importance cardinale : la littérature orale, la mythologie, la musicologie, l'esthétique picturale et architecturale, la critique littéraire etc. Cette multiplicité d'usages signe une valeur heuristique certaine et ne manque pas ailleurs de soulever des questions de postée épistémologique.

Les travaux d'un ethnologue comme Propp ', d'esthéticiens et de narratologues comme Brémond ${ }^{2}$ et Greimas ${ }^{3}$ abordent frontalement le terme du motif qui renvoie, en réalité, à la question fondamentale du sens (celui des œuvres, des discours et des cultures) : si le motif oriente, détermine, construit du sens, le motif est également sens.

Dans son ouvrage consacré à la Morphologie des contes, Propp oppose une conception de la divisibilité des motifs (mais aussi des sujets) aux conceptions atomistes de ses prédécesseurs pour qui "le motif ou le sujet dans son ensemble étaient considérés comme des monades narratives indécomposables ${ }^{4}$. De son côté, Thierry Charnay précise que le mode du motif n'est pas celui d'une unité discursive réalisée mais "d'une virtualité inscrite dans une sorte de mémoire transtextuelle"s.

Relations entre le sujet et le motif, invariance/variance pour le premier, transtextualité pour le second, on voit combien la portée de la réflexion sur le motif est déterminante, d'autant plus que celui-ci bénéficie d'un statut bivalent : il se pose en objet de connaissance et se définit en même temps comme support d'une stratégie descriptive. De plus, le fait d'une circulation des motifs non seulement transempirique, transculturelle, mais aussi intraculturelle, présente un intérêt anthropologique évident.

L'approche anthropologique du motif se fonde sur les points majeurs suivants :

- le motif peut être considéré comme une unité discursive ;

- le motif peut avoir une représentation matérielle (aspects d'une architecture, par exemple) ou immatérielle (un mot) ;

- le motif s'inscrit dans une histoire sociale et culturelle qui explique sa formation, sa diffusion, sa circulation, éventuellement son déclin;

- le motif agit en méta-niveau de discours dans la mesure où il renvoie à un "ailleurs" qui peut relever d'un invariant culturel. 
Le processus de singularisation culurelle de la société basque, au $\mathrm{XX}^{e}$ siècle, appuyé sur la formation d'une double rhétorique - patrimoniale et politique conduit à une multiplication de motifs. L'enquête sur le néo-style architectural basque, apporte de précieux enseignements pour l'étude d'un type particulier de motif : le mode d'appellation.

L'histoire du motif linguistique dans l'habitat est intimement liée à l'histoire de la formation et de la diffusion du néo-style basque sur l'espace urbain côtier du Pays basque, néo-style basque dont nous avons analysé précédemment les caractères anthropologiques ${ }^{6}$. Ce type de motif, qui accompagne la naissance de la "Villa" basque à la fin du XIX e siècle, marque un déplacement à l'égard des mécanismes de la reproduction sociale au sein de la société rurale basque où le nom de la maison (de caractère toponymique) servait de moyen d'identification sociale de l'individu, et la naissance de l'usage esthétique, décoratif de l'appellation basque de la résidence. Espace social en voie de constitution et en même temps lieu d'invention de cette nouvelle pratique culturelle, tel s'impose l'espace urbain côtier dans le dernier quart du XIX ${ }^{e}$ siècle.

\section{A. Invention bourgeoise et fonction décorative}

La formation de I'armature urbaine de la Côte Basque (Bayonne mise à part) est due essentiellement aux conséquences sur le plan touristique des différents séjours de la famille impériale napoléonienne à Biarritz, ville érigée en lieu de villégiature très recherché par une clientèle aisée de la région parisienne. Cette structuration sociologique locale se manifeste dans des formes architecturales caractérisées par une variété et une audace impressionnantes des sty'les, et résumées dans le nouvel objet architectural qu'est la "Villa" : des villas d'inspiration néo-gothique ou espagnole aux villas bretonnes et basques. Symbole ${ }^{7}$ marquant d'une bourgeoisie ayant accédé à une opulence digne d'être remarquée, la "villa" condense et met en scène de multiples signes et sens : l'imaginaire social d'une classe sociale triomphaliste, les jeux culturels autour des styles (historiques et régionalistes/populaires), les essais de modélisation d'une "modernité" architecturale contre un classicisme architectural usé mais aussi contre des mentalités "modernistes" regrettables.

"Le Pays basque a été mis en vedette, glorifié, chanté, par des littérateurs et poètes au début du véritable essor touristique. Il a été traversé, parcouru, admiré, quand l'automobilisme entraîna ce grand courant touristique. Et l'enthousiasme accompagna la révélation. Ce coin des Pyrénées à l'extrémité de la France, trop longtemps ignoré par le Français, a reçu l'enviable hommage d'être reconnu comme l'un des plus jolis coins de la "Belle France". Désormais, sa renommée est établie et sa fortune assurée. Elle s'affirme d'année en année. Et, bien entendu, l'aisance a créé des besoins nouveaux. Le splendide isolement ancestral ne peut être. Les mours ont évolué ainsi que les usages traditionnels des autochtones conservés jusqu'à ce jour presque intacts. Ainsi, la vie sociale a changé, les mœurs champêtres également, mais il y a encore la vétuste métairie des aïeux, réparée, rajeunie, blanchie à neuf. Et non loin, souvent juxtaposée, il y a la jeune sœur contemporaine, sœur élégante qui n'a pas toujours la grâce rustique de l'ancienne et aussi la simplicité qui lui conviendrait beaucoup mieux. Elle oppose même un certain air altier, l'air de prospérité cossue, inévitable, du reste, chez une richissime parente. Pour tout dire, la villa basque moderne est une création parmi 
les essais régionalistes les mieux réussis", note H. Godbarge, fin interprète de l'architecture "régionaliste et moderne" basque ${ }^{8}$.

Dans un paysage architectural, tant urbain que rural, figé depuis plusieurs siècles, la villa néo-labourdine (s'inspirant du style architectural de la province côtière du Labourd, fait notamment de colombages et de murs de refend organisant la façade, de toit à pentes souvent dissymétriques, etc.) baptisée "villa basque" constitue la grande invention architecturale du XIX ${ }^{\circ}$ siècle portée par une pléiade d'architectes ${ }^{9}$ séduits par toutes les virtualités d'une architecture moderne de version régionaliste, et correspondant aux attentes sociales et culturelles d'une grande bourgeoisie, étrangère à la région, éprise de pittoresque et d'exotisme, trouvant dans le spectacle de l'architecture populaire basque de nouvelles opportunités de distinction sociale.

A la fois entité architecturale urbaine et objet socialement distinctif, la villa néo-basque apparaît comme le produit d'une double distanciation géographique et culturelle - à l'égard d'une société rurale/agricole travaillée par une logique communautaire selon laquelle la seule déclinaison de l'appartenance à un etxe suffit au repérage social d'un individu; cette logique communautaire ignorant la définition administrative de l'individu imposée par l'État républicain.

Conformément à cette logique, le nom de la maison, construit principalement sur une base toponymique, est un attribut anthropologique fondamental de continuité historique ; ainsi, il transcende la discontinuité entre les générations, imprimant au paysage social une forte apparence d'immuabilité. En occupant une telle place dans les mécanismes de reproduction communautaire, le nom de la maison y apparait comme un motif à forte puissance anthropologique.

Tels ne sont pas le statut et le destin social et historique du nom de la villa néobasque dont l'édification repose, nous l'avons vu, simultanément sur un transfert (de signes) et un détournement de sens; ce dernier constat est particulièrement sensible avec la constitution d'un corpus de termes nouveaux pour désigner la villa néo-basque : le motif linguistique à usage décoratif pour l'habitat est né.

Toki-Eder ("Bel endroit"), Haitzurra ("Pioche"), Harotzaldea ("Du côté des étrangers", Guétary, 1900), Etche Pherdea ("Maison verte", Guéthary, 1905). seront parmi les premiers motifs (dont certains, tel Toki-Eder, fondateurs de véritables lignées) de ce qui deviendra une forte habitude culturelle. Le motif linguistique n'existe et ne se comprend que par rapport à la "Villa", totalité significative soumise aux impératifs de la décoration et de la représentation (ce qui n'écarte nullement la recherche de la fonctionnalité). Le mur de refend, le faux colombage, la dissymétrie voulue du toit, le nom basque, etc. constituent autant de segments équivalents et indissociables de cette totalité.

Ce type de motif marque une rupture avec le nom de la maison paysanne basque dont on a déjà évoqué le contenu à dominante toponymique. La forme et le fond reproduisent et mettent en scène le goût du singulier qui recouvre, ici, le pittoresque, l'inattendu, l'insolite. Tous les artifices sont utilisés - ainsi la sonorité de la langue basque avec Haitzurra - pour accentuer l'originalité du motif.

De cette apologie du singulier se nourrissent la distinction bourgeoise de même que la rhétorique esthétique qui la fonde et qui englobe l'extérieur et l'intérieur de la villa basque. Si l'un et l'autre sont régis selon les deux modalités 
notées par J. Baudrillard ${ }^{10}$ (analysant l'ordre esthétique bourgeois), à savoir les principes de saturation et de redondance d'une part, de symétrie et de hiérarchie d'autre part, seul l'extérieur - et en particulier la façade principale - se prête aux multiples jeux culturels autour du modèle paysan originel et paraît autoriser la définition néo-basque de l'habitation, - l'intérieur, c'est-à-dire la décoration, l'ameublement et l'agencement des pièces, obéissant aux règles de l'esthétique bourgeoise et d'une fonctionnalité qui se veut "moderne".

Cette division entre "l'intérieur" et "l'extérieur", repérable également dans le motif entre une syntaxe basque et une sémantique sans ligne directrice si ce n'est celle de la fantaisie, rappelle la distinction théorique proposée par $\mathrm{R}$. Boudon " entre le système qui désignerait les faits de surface et la structure qui rendrait compte des faits latents; on aurait ainsi un "système du néo-basque" (agencement des formes et des volumes) et une "structure du néo-basque" (détournements et errements de sens). A cette approche a-historique, nous préférons inscrire la villa néo-basque dans l'historicité, c'est-à-dire voir en elle une illustration (exemplaire?) du système d'action d'une classe bourgeoise obsédée par l'affirmation de sa visibilité sociale et un système d'analyse impliquant la représentation d'une pluralité de valeurs matérielles et symboliques : bourgeoises et populaires/paysannes urbaines et rurales, basques et françaises, traditionnelles et modernes. Si la villa néo-basque est, par sa somptuosité, un objet architectural inaccessible pour le "peuple", elle tend cependant à neutraliser les tensions, toujours fortes, entre la Tradition et la Modernité. Cuvre d'une bourgeoisie triomphaliste, la villa néo-basque signe-t-elle pour autant une "modernité triomphale" pour reprendre une formule d'A. Touraine ${ }^{12}$ ? Non, car elle représente l'essai de modélisation culturelle la plus audacieuse d'une nouvelle modernité fondée sur l'association active des exigences de la raison (manifestes dans la recherche de la fonctionnalité et de la conformité à la modernité) et les désirs de l'individu déclinés en termes de sensibilité au charme et à la fantaisie.

A cet égard, le motif linguistique résume sans doute le mieux cette sorte de gratuité culturelle que signent les errements de sens notés (nommer en effet une villa : "pioche", "petite montagne", etc.) et qui anime l'imaginaire du résident pavillonnaire bourgeois.

La seconde moitié du XIX ${ }^{e}$ siècle se distingue, on le sait, par la concomitance et l'hypertrophie de deux discours sur le sens de l'histoire; l'un sur la modernité dont le domaine de gestation est la ville, l'autre sur les effets de cette modernité, et en particulier sur l'appauvrissement social et culturel d'un monde rural dépositaire de toutes les vertus. C'est dans le sillage de ce regard que la pensée ethnologique prendra forme.

La villa de néo-style régional naît à l'intersection de ces deux discours : si la modernité s'empare du gothique (expression conjointe d'une temporalité et d'un art) pour produire du néo-gothique dont l'écho culturel restera très limité, c'est avec les styles régionaux qu'elle prouvera sa plus grande fécondité. Bien entendu, cette fécondation n'aurait pu avoir lieu sans le regard culturel de cette bourgeoisie urbaine exogène, regard à la fois ambivalent et ambigu, "s'intéressant" aux aspects les plus visibles de la culture matérielle, c'est-à-dire l'architecture comme à la société la plus archaïsante, c'est-à-dire la paysannerie, pour sa propre mise en scène sociale. 
Le faible succès du néo-gothique, en particulier, et la faveur très grande dont jouit le néo-style régional peuvent laisser à penser que les écarts entre classes sociales sont plus propices à un traitement culturel et idéologique que les écarts entre périodes historiques.

Ainsi, la formation de motif linguistique comme mode d'appellation de la villa basque s'apparente, dans un cadre décontextualisé géographiquement, à la première mise en scène sociale de la langue basque (plus précisément, de mots de cette langue) dans une perspective qualifiable d'instrumentale. Celle-ci ne signifie nullement la valorisation de la langue basque comme vecteur de communication sociale mais plutôt la réduction de la langue à un fonds patrimonial spécifique dans lequel chacun puise selon sa fantaisie. Aussi cette forme inédite d'utilitarisme social du basque nous apparaît-elle comme la première manifestation locale de ce mouvement culturel qu'est la patrimonialisation ${ }^{13}$ et auquel les décennies suivantes donneront un écho croissant.

\section{B. La dispersion sociale et géographique des motifs linguistiques}

La diffusion du néo-style basque tout au long du $\mathrm{XX}^{\mathrm{e}}$ siècle s'opère selon deux modalités. L'une sociale : cette production architecturale bourgeoise séduit progressivement d'autres fractions sociales jusqu'à devenir un banal objet de consommation. L'autre, géographique, du Pays basque côtier, le style néo-basque se diffuse vers l'intérieur du pays mais aussi dans l'ensemble du Sud-Ouest jusqu'au midi toulousain. Ce processus répond, comme cela a été souligné, aux transformations sociales majeures de ce $\mathrm{XX}^{\mathrm{e}}$ siècle que sont, en particulier, l'élargissement des classes moyennes, le rôle décisif d'une mobilité sociale due pour l'essentiel à la capitalisation culturelle, le renforcement de l'attractivité urbaine. L'histoire des objets obéit tout à fait logiquement à ces transformations. Ainsi, sur le plan architectural, à côté de la villa, se constitue une nouvelle figure d'habitat, le pavillon, dont on connaît le succès à partir de la dernière guerre mondiale et la teneur idéologique dans l'imaginaire individuel contemporain.

Le développement conjoint de l'idéologie pavillonnaire et d'une économie du logement entraîne la diversification des modes de construction et de promotion de l'habitat. Outre les architectes, les maçons, mais aussi les documents publicitaires, interviennent désormais dans la définition des options architecturales. Traitant de la place du néo-basque à Toulouse, Paulette Girard et Thierry Mandoul notent que "Toulouse paraît avoir une fascination pour le Pays basque ; villégiature, sport, pêche vont lui être associés. Si ce mouvement commence avant la guerre, il connaîtra une accélération décisive après la loi des congés payés du Front Populaire. Ainsi, les publicités et réclames vont fleurir pour passer vacances et week-ends au Pays basque, en témoignent celles recueillies dans les bulletins municipaux de 1936 et 1937 . Il semble que Toulouse présente cette particularité d'avoir été, comme la vague néo-basque durant l'entre-deux guerres hors la Côte d'Argent ou les Landes, le troisième site du néo-basque de cette période" ${ }^{14}$. Cependant, le néo-style régional et basque en particulier n'est plus exempt de dérives néfastes qui le déconsidèrent, ainsi que le souligne la revue l'Art Méridional: "On ne veut pas parler ici, bien entendu du faux régionalisme qui consiste à prendre les façades d'une ferme pour en faire une somptueuse villa, comme Rostand à Arnaga (Cambo-les-Bains - Pays Basque) ou tant d'autres sur la Côte d'Azur. Laissons ces fantaisies au plateau de l'opéra comique. Le régio- 
nalisme, le vrai, est une chose bien plus subtile. Il ne tient pas plus à la répétition d'un ornement que le style Henri II aux initiales enlacées du roi et de Diane de Poitiers. Il faut, pour le déceler, une étude attentive des atavismes, une comparaison minutieuse des mouvements du passé et de nos jours" ${ }^{15}$. Mais qu'advient-il du motif linguistique dans cette effervescence culturelle qui porte le style néobasque?

Les nouvelles hiérarchies qui signent les options architecturales - villa, pavillon, maison "clé en mains" - et la dispersion sociale et géographique du modèle néo-basque n'entraînent pas la formation de corpus linguistiques propres aux classes sociales qui l'adoptent. Aux motifs du début du XX $\mathrm{XX}^{\mathrm{e}}$ siècle, font écho les motifs des villas construites entre les années vingt et quarante : Irrintzina ("cri des Basques", Ciboure 1929), Asmuteguia ("Lieu des pensées", Saint-Jean-deLuz, 1922) Pare Gabea ("Sans équivalent", Saint-Jean-de-Luz, 1923) Arri Gorri ("Pierre rouge", Bayonne, 1926), et aussi les motifs des habitations édifiées depuis 1950 dont la seule ville d'Ustaritz (cité en pleine expansion résidentielle et située dans la périphérie de Bayonne) fournit un échantillon bien significatif : Gure doya ("Ce qui nous suffit"), Gure egoitza ("Notre demeure"), Iguski Alde ("Face au soleil"), Bichta Eder ("Belle vue"), Goiseko Izarra ("Étoile du matin"), Zoriontsu ("Heureux"), Nic nahia ("Ce que j'ai voulu"), Gostukoa ("Qui convient"), Gure Lanetik ("De notre travail"), Gure xokoa ("Notre coin"), Goxoki ("Agréablement"), Agur ("Bonjour"), Argi eder ("Belle lumière"), Gure etxola ("Notre borde"), Gure izarra ("Notre étoile"), Aire ona ("Bel air"), etc.

Si l'idéologie pavillonnaire a étendu et conforté l'usage du motif linguistique basque, elle a intégré localement les conformismes ou les mimétismes constatés ailleurs, de multiples fois, par les recherches en sciences sociales (du type "Ca me suffit", "Bel air", "Mon désir", etc.). Alors que la classe sociale utilisatrice du premier néo-style basque était exogène, la formule pavillonnaire séduit les populations locales confrontées à une mobilité géographique (de la campagne vers la ville) et sociale qui comporte une évaluation plus ou moins réfléchie des risques, des bénéfices et des obligations liées à cette mobilité. Parmi les données soumises à cette évaluation figurent les usages linguistiques. Ceux pour qui la mobilité sociale a supposé l'affranchissement de leur passé culturel, ont abandonné l'usage du basque comme langue de la communication sociale, la conservation de cet élément résiduel - le motif - entretient le sentiment d'une continuité culturelle entre la condition de départ et le statut atteint. Pour les individus extérieurs au Pays basque, le recours à ce motif basque affiche une intention d'ancrage social local, exercice sans doute facile mais néanmoins à forte charge symbolique, révélant une autre fonction, celle de la médiation. Et cette fonction médiatrice est à inscrire dans l'ordre des possibilités culturelles issues du processus de patrimonialisation dont nous analysons, dans les lignes suivantes, les dernières ruses.

\section{Ultimes jeux}

Jusqu'à une période récente, le motif linguistique basque a été l'apanage exclusif de l'habitat individuel. A cela des raisons d'ordre statistique et sociologique : le résidentiel individuel a connu un développement nettement supérieur au résidentiel collectif et les processus d'individualisation sociale à l'œurvre dans la société moderne privilégient l'option pavillonnaire. L'observation des modes d'appellation des immeubles collectifs construits lors de cette dernière décennie 
met en évidence l'introduction du motif linguistique basque dans les résidences collectives de luxe et l'élargissement du corpus linguistique par l'introduction de termes inédits. Comment ne pas établir une correspondance entre la grande villa

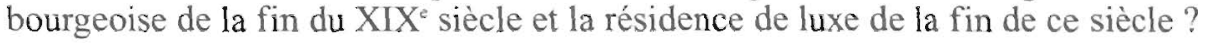
Troublante homologie structurelle. Résidences Eskualduna ("Le basque", SaintJean-de-Luz), Eskuara ("La langue basque", Guéthary), Amaya ("Prénom basque moderne", Biarritz), Kantaldi ("Tour de chant", Guéthary), Euskadi ("Nom des territoires basques autonomes d'Espagne", Biarritz) : la recherche décorative autorise, ici, toutes les audaces sémiques, jusqu'aux emprunts de termes liés à l'histoire de la société basque contemporaine et ayant une forte connotation politique. Nouveaux errements de sens ou domestication de sens rebelle, le motif de la résidence de luxe informe sur la plasticité culturelle de l'imaginaire contemporain. Au même moment, la puissance du sens patrimonial se confirme, manifestant ses capacités intégratrices. Pour la résidence collective dépourvue de toute référence architecturale néo-stylistique basque, le motif linguistique constitue l'indice élémentaire de la référence à l'environnement basque. Sa portée symbolique en est d'autant plus accusée. Le processus de socialisation du motif basque se veut totalisant, affectant progressivement tous les modes résidentiels de manière chronologique (la villa, le pavillon, la résidence collective de grand standing ; manque pour le moment la résidence collective "populaire") jusqu'à inclure les lotissements, infrastructures déterminant les contours de la socialibilité du futur quartier.

En disant cela, il ne s'agit pas de chercher au sein de la société basque ellemême le principe explicatif de cette inventivité. Portée initialement par les classes bourgeoises, elle a tendance à se disperser aujourd'hui au sein de la société moderne et à s'incarner dans les circonvolutions d'une idéologie patrimoniale aussi séduisante qu'efficace.

Pierre BIDART UPPA 


\section{Notes}

1. V. Proop, Morphologie du conte, Paris, Ed. du Seuil, 1965 et 1970, 254 p., voir en particulier, p. 21 et suivantes.

2. Cl. Brémond, La logique du récit, Seuil, 1970, 254 p.

3. A.J. Greimas, Du sens, Paris, 1970.

4. V. Propp, ouv cité, voir l'article de Evguéni Mélétinski sur "L'étude structurale et typologique du conte".

5. Voir Th. Charnay, "Quel motif?", dans Ethnologie Française, 1995/2, p. 149-151, p. 151.

6. Voir notre étude, "Le néo-style basque comme objet anthropologique" dans Ethnologie Française, XXV, 1995, 4, p. 681-685.

7. A bien des égards, la villa néo-basque originelle appartient au domaine des habitudes culturelles de la "dépense ostentatoire" ("Conspicuous Waste") analysées par Thornstein Veblen dans son célèbre ouvrage, The theory of the leisure Class (1899), dont les Editions Gallimard ont publié la traduction française, La théorie de la classe de loisir (1969). La présence d'une domesticité nombreuse et les caractères de somptuosité et d'immensité des résidences confortent cette observation.

8. H. Godbarge, Les Arts basques anciens et modernes, Hossegor, Chabas, 1931, p. 89. Voir aussi "Architecture Régionale : l'architecture en Pays basque" in l'Architecture, Vol. XLIX, n 3, I, 5 mars 1931, p. $73-83$

9. La longueur de cette liste, reproduite dans le chapitre antérieur, souligne l'écho du thème architectural dans les débats suscités par le régionalisme. Sans doute parce que l'architecture permet, plus que tout autre art, de traduire et de réconcilier les dimensions du passé et du présent.

10. Voir J. Baudrillard, Pour une critique de l'économie politique du signe, Gallimard, 1972, p. 25.

11. R. Boudon, A quoi sert la notion de structure, Paris, Gallimard, 1968.

12. A. Touraine, Critique de la Modernité, Fayard, 1992.

13. Voir P. Bidart, "Patrimoinc et Ordo rerum dans les sociétés modernes", dans Ethnologie française, XXVII, 1997-2, p. 244-251.

14. P. Girard et Th. Mandoul, "L'architecture néo-basque à Toulouse (1920-1940)", dans Architectes et architectures des Pays basques $\left(X I X^{\prime}\right.$ - $X X^{*}$ s.). sous la direction de P. Bidart, Ed. Izpegi, 1997, p. 49.

15. Toulouse, la ville et ses architectes (1920-1940), Éditions Ombres, 199 1, p. 72 